\title{
Fundamental Issues in Manufacturing Photovoltaic Modules Beyond the Current Generation of Materials
}

\author{
G. F. Alapatt, R. Singh, and K. F. Poole \\ Holcombe Department of Electrical and Computer Engineering and Center for Silicon Nanoelectronics, Clemson University, \\ Clemson, SC 29634-0915, USA \\ Correspondence should be addressed to R. Singh, srajend@clemson.edu
}

Received 30 April 2011; Accepted 27 September 2011

Academic Editor: Surya Prakash Singh

Copyright ( 2012 G. F. Alapatt et al. This is an open access article distributed under the Creative Commons Attribution License, which permits unrestricted use, distribution, and reproduction in any medium, provided the original work is properly cited.

\begin{abstract}
Many methods to improve the solar cell's efficiency beyond current generation of bulk and thin film of photovoltaic (PV) devices have been reported during the last five decades. Concepts such as multiple exciton generations (MEG), carrier multiplication $(\mathrm{CM})$, hot carrier extraction, and intermediate band solar cells have fundamental flaws, and there is no experimental evidence of fabricating practical higher efficiency solar cells based on the proposed concepts. To take advantages of quantum features of nanostructures for higher performance PV devices, self-assembly-based bottom-up processing techniques are not suitable for manufacturing due to inherent problems of variability, defects, reliability, and yield. For processing nanostructures, new techniques need to be invented with the features of critical dimensional control, structural homogeneity, and lower cost of ownership as compared to the processing tools used in current generations of bulk and thin-film solar cells.
\end{abstract}

\section{Introduction}

Starting with providing power to first communication satellite, Telstar, in 1962, photovoltaic (PV) systems have evolved to a market size of about $16 \mathrm{GW}$ in 2010. Current commercial PV market is based on bulk solar cells ( $\mathrm{Si}$ and III-V compound semiconductors) and thin-film solar cells based on a-Si, CdTe, and CuInGaSe2, while the devices are based on p-n homo- and heterojunctions and tandem junction solar cells. In case of III-V compound semiconductor-based concentration solar cells, one sun efficiency of $35.8 \%$ has been achieved [1]. Being an active area of research, new materials and structures are constantly being investigated in the hope of getting efficiencies higher than the typical bulk silicon solar cell efficiency of about $20 \%$. Solar cells, which can be possibly made from engineered materials and nanostructures, are often called "third-generation PV cells" [2], with the materials themselves being referred to as "next generation materials," "smart materials," or "intelligent materials." In reality, despite the semantics and the buzz, there has been no improvement in the efficiency of a solar cell using the so called "smart materials." Of course, with better engineering, conventional bulk and thin-film solar cells have become more reliable, their cost has decreased over the years, and their efficiencies have also increased by a small percentage. However, this increase in efficiency cannot be attributed to any nanostructured smart material. This raises the interesting first question-during the last 10 years, why has there been no significant increase in the "third-generation" solar cell efficiency even with the introduction of "smart materials"? The second most important question one would like to know is if there are any fundamental barriers that cannot be surmounted by any technological advancement. The objective of this paper is to examine the "third-generation" solar cells from the manufacturing point of view and answer the two questions raised previously.

\section{Manufacturing Requirements of Photovoltaic Modules}

Irrespective of the type of materials used in the manufacturing of PV modules, the following key criteria are used in selecting an appropriate technology: (i) no material supply constraint, (ii) low cost of ownership, (iii) low production cost, (iv) prospects of further cost reduction, and (v) green 
manufacturing with no environmental safety and health issues. Even for silicon (dominant photovoltaic material), the question of supply chain is very important. Silver is used in the manufacturing of bulk silicon solar cells and is being replaced with copper [3]. As shown in Figure 1 [3], silver's prices have increased significantly in the last 5 years. Due to the use of silver by the film, battery, and the electronic industry, the supply chain of silver also affects the PV industry [3]. Such trends will happen often in the future, and the manufacturing technology should be able to withstand the swings of the market trends. In previous publications, we have discussed the detailed economic requirements of manufacturing photovoltaic materials and PV systems and the interested reader is referred to [4-6].

\section{Next Generation Materials}

Next generation materials for solar cells include any material or material structure that is currently not being mass produced to manufacture solar cells. This term also refers to organic materials and dyes that are used to fabricate organic solar cells and dye sensitized solar cells. In addition, this term also includes nanostructure materials processed using unproven solar cell manufacturing technologies such as selfassembly. These topics are explained in this section.

3.1. Organic- and Dye-Sensitized Solar Cells. These cells are often processed in a liquid form, with the hope of making them cheaper than current commercial solar cells. Reasonable efficiencies have been achieved using organic materials such as PCBM (phenyl-C61-butyric acid methyl ester). According to [7], the best values of efficiency of organic solar cell and organic submodule are $8.3 \%$ (device area = $1.031 \mathrm{~cm}^{2}$ ) and $3.5 \%$ (aperture area $=308.4 \mathrm{~cm}^{2}$ ), respectively. This lower efficiency is a direct result of the lower electron mobility, poor contact to electrodes, and defects in the material. Apart from these problems, organic solar cells have reliability issues and are degraded when exposed to sunlight and air $[8,9]$.

According to [7], in case of dye-sensitized solar cells, the device and submodule efficiencies are 10.9\% (device area $=$ $1.008 \mathrm{~cm}^{2}$ ) and $9.9 \%$ (aperture area $=17.11 \mathrm{~cm}^{2}$ ), respectively [7]. Dye sensitized cells share many advantages and disadvantages of the organic solar cells. Some of the similarities are hope of low cost for processing, low efficiency, high concentration of defects, and reliability problems. In both types of solar cells, low carrier mobility because of structural inhomogeneities and the lack of an ordered structure is the fundamental problem. Without any fundamental breakthrough of inventing new organic and dye-sensitized photovoltaic materials, these solar cells will not play a significant role in large-scale utilization of PV for power generation. However, these solar cells have the advantage of being flexible and light and can provide power for a niche market consisting of short-lifetime products such as clothing, bags, and temporary shelters.

3.2. Nanostructured Materials. Using nanomaterials, many concepts have been proposed to increase solar cell efficiency.
Examples include multiple electron generation (MEG), carrier multiplication (CM), hot carrier extraction, and intermediate band solar cell. These concepts rely on the performance of nanomaterials for obtaining high efficiency. Quantum dots, a particular nanostructure, have been proposed to increase the solar cell efficiency. To understand nanostructure-based solar cells, fundamentals of nanostructures are presented in the next section.

\section{Fundamentals of Nanostructures}

Properties of materials are different when the material's dimensions are only a few nanometers. Such properties can be used to bring enhancements to the solar energy conversion efficiency, if such materials can be successfully manufactured. Currently, commercial solar cells are made from bulk or thinfilm materials, or a combination of materials, without taking into consideration properties such as quantum confinement that are associated with a dimension of less than about $10 \mathrm{~nm}$. In the past few decades, there have been proposals of harnessing quantum-confinement-related properties of materials to yield devices such as high-efficiency solar cells and faster transistors. Many of the proposed concepts capitalize on using the properties which arise from the quantization of energy, momentum, and density of states in materials at very small dimensions. For instance, a spherical particle with diameter of about $5 \mathrm{~nm}$ will exhibit remarkably different properties than a spherical particle of the same material with a diameter of $1 \mathrm{~mm}$. As early as 1976, Buffat and Borel [10] showed that the melting point of gold particles decreases by about 100 degrees Kelvin as its diameter is made less than $100 \mathrm{~nm}$. Such properties can be generalized, and it is observed that all material properties will undergo changes as the material dimension becomes less than a few nanometers [11]. This concept is illustrated in Figure 2.

The next generation materials that exhibit immensely different properties when compared to regular PV materials can be broadly classified into four categories: $1 \mathrm{D}, 2 \mathrm{D}, 3 \mathrm{D}$, and $0 \mathrm{D}$. The first classification, $1 \mathrm{D}$, refers to structures that have only one dimension significantly larger than the other two dimensions. Examples of such a structure include chains or bundles of molecules or polymers. $2 \mathrm{D}$ nanostructures have two dimensions that are significantly larger than the third nanodimension; examples of such a structure are films of nanomaterials that are only a few nanometers in thickness, but may have much larger widths and lengths. $3 \mathrm{D}$ nanostructures include honeycomb-like structures or a matrix of particles formed by the aggregation of nanoparticles. In such a structure, none of the dimension may be in the nano range. Finally, in a $0 \mathrm{D}$ structure, all the dimensions are only nanometers wide, and, consequently, it has none of its dimensions that are larger than a few nanometers [12]. In all these classes, quantum confinement brings about a change in the material properties. The quantization happens in different dimensions in all the classes of materials. To illustrate this point, in the $0 \mathrm{D}$ structure, all dimensions are only a few nanometers wide and the density of states in that structure is quantized in all three dimensions, while, in the $2 \mathrm{D}$ structure, only 1 dimension is on the nanoscale range, and thus, the density 


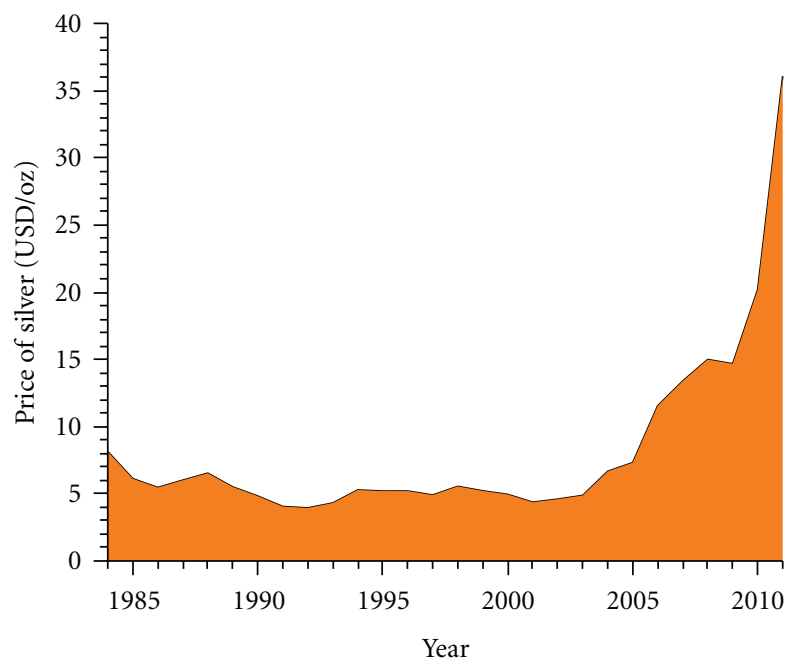

FIgURE 1: Prices of silver in the last 25 years.

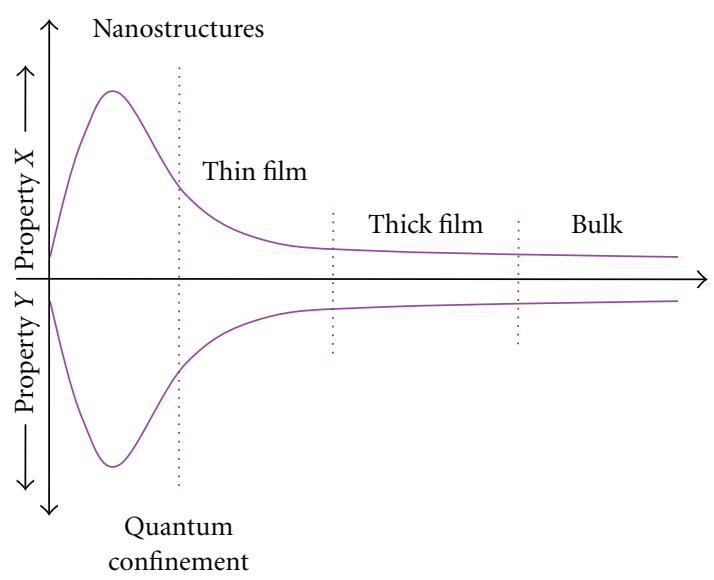

FIGURE 2: Change of properties with dimension ranging from one atom to bulk dimension [11].

of states is quantized only in one dimension. Figure 3 [13] shows the energy states due to quantization in these structures.

As stated earlier the interesting and sometimes useful properties of nanostructured devices arise due to the quantum confinement effect. This has been understood for many years; however, obtaining experimental results to compare with the theoretical model is difficult because of defects and the presence of large surface states, which are present because the ratio of states on the surface of the nanomaterial is higher compared to that present in the interior of the nanomaterial. One should have a good control and understanding of the defects and surface states to be able to make use of nanostructured materials. This is the reason why researchers have been able to find interesting properties in an isolated nanostructure, but not always in a device made from the nanostructure. There has been numerous attempts to use nanomaterials (fabricated by nonlithography techniques) in a variety of applications such as transistors, metallic interconnects, dielectrics, and diodes. Even though many principles remain the same across a wide range of applications, this paper will focus specifically on the application of nanomaterials to create the active material of a solar cell, the term "active" is used specifically to differentiate the actual solar cell material that converts light energy into electrical energy from other parts of a solar cell such as electrodes, antireflection coatings, and interconnects.

In 1961, Shockley and Queisser calculated the theoretical limit of solar energy to electrical energy conversion for a single junction silicon solar cell as 33\% [14]. Until now, this limit has not been exceeded experimentally, the highest efficiency reported in silicon solar cells being only 25\% [7]. There have been numerous articles of the possibility of obtaining efficiency much higher than $33 \%$ in single junction solar cells by using techniques such as MEG, intermediate band solar cell (IBSC), and hot carrier solar cell [15-19]. The earliest of these concepts, the IBSC, was proposed in 1960 by Wolf [20], while the concept of extracting electron hole pairs of energy higher than the band gap of the material was proposed in 1982 [21]. To fabricate such high-efficiency solar cells, researchers often propose using nanosystems such as nanodots, nanowires, nanocrystals (NC), and carbon nanotubes [19, 21-24]. Since the 1960s, numerous experiments were conducted to fabricate such high-efficiency cells and none of them was successful. This failure to obtain a higher efficiency even with many years of research points us towards the fundamental flaw of such proposed concepts.

The fundamental flaw in the MEG concept is that the indirect measurement of photoluminescence and related experimental work relates only to local generation of photocarriers and has no relevance to the transport of generated photocarriers over the barrier of the junction. Research towards obtaining a high-efficiency solar cell does not end with the observation of the generation of electron hole pairs (EHPs); these created EHPs must also be able to move to the electrodes without interacting significantly with the surroundings. The transport involves carrier-phonon and carrier-lattice interactions, which are minimum in pure structures such as an ultrapure crystalline silicon solar cell. Only an electrical measurement involving measurement of short-circuit 

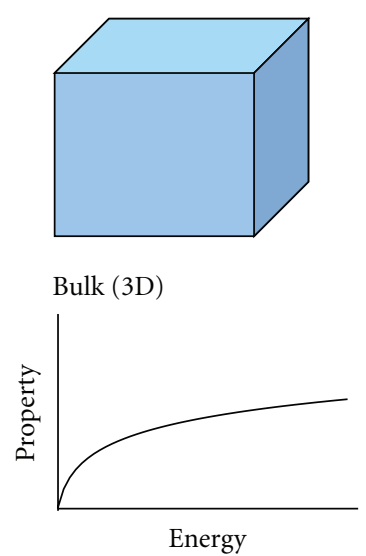

(a)
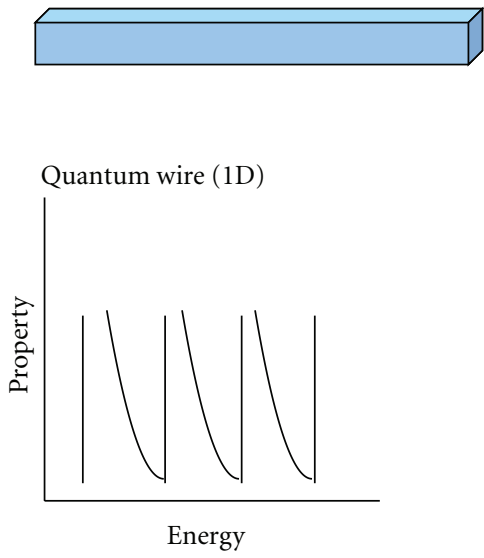

(c)
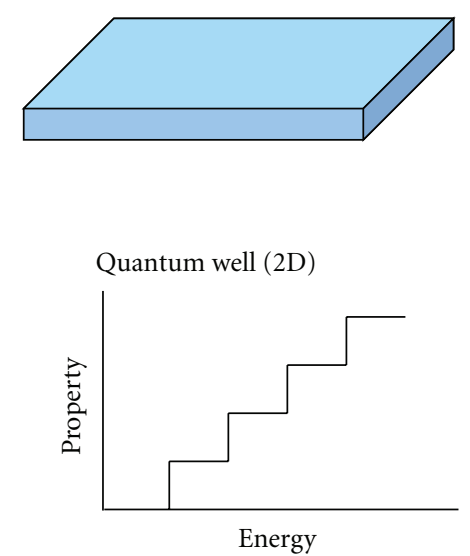

(b)
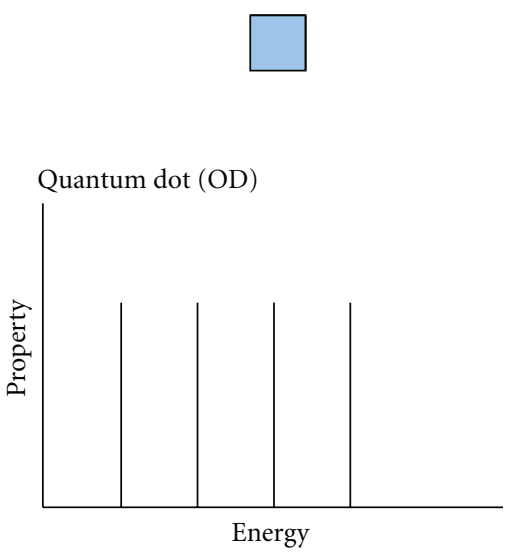

(d)

FIGURE 3: Quantization of properties with scaling of dimensions [13].

current and open-circuit voltage reveals how efficiently a cell can convert the photoenergy to electrical energy. Unless this test is performed, one cannot speculate about the efficiency. From a manufacturing point of view, this test must be performed on sufficient number of large area samples to account for process variability and defects.

\section{Quantum Dots}

Quantum dots are "0" D particles, in which the band structure is dependent on the particle size because of quantization effects. In theory, the band gap can be controlled by varying the particle size and one can engineer an optimal band gap for high-efficiency solar energy collection. In addition, other methods such as multiple exciton generation (MEG) and hot carrier capture processes have been postulated to increase the solar energy conversion efficiency. Quantum dot proof of concepts for use as solar cells is generally based on transient absorption spectroscopy experiments, in which the number of carriers generated are counted using light sources and detectors. Figure 4 [25] shows a typical decay curve, which shows that carriers were generated at zero picoseconds (ps) and that the carrier population decayed over a time of 400 ps. This does show that carriers were generated, but these high-energy carriers were not transported spatially across a barrier. The transport process across the barrier must take place before these high-energy carriers can be used for generating electricity. To date there is no experimental data in quantum dots that shows the high-energy carrier transport across the barrier takes place in a quantum dot.

In an experiment conducted in 2009, researchers fabricated a quantum dot photodetector, which is very similar to the fabrication of a solar cell. As shown in Figure 5, external quantum efficiency (EQE) was measured and the number varied between 15 and $52 \%$ as the incident wavelength was varied between 400 and $1800 \mathrm{~nm}$ [26]. This is the realistic quantum efficiency with which the incident photon is able to generate measurable carriers in a colloid of quantum dots. Some of the photogenerated carriers have recombined in quantum dots, and the number of collected carriers per incident photon is less than $100 \%$ for all wavelengths.

\section{Processing of Nanostructures}

To manufacture a nanostructured device in a commercially viable fashion, two technologies are currently under consideration. The first one is the standard top-down approach, and the other is bottom-up approach. The most important 


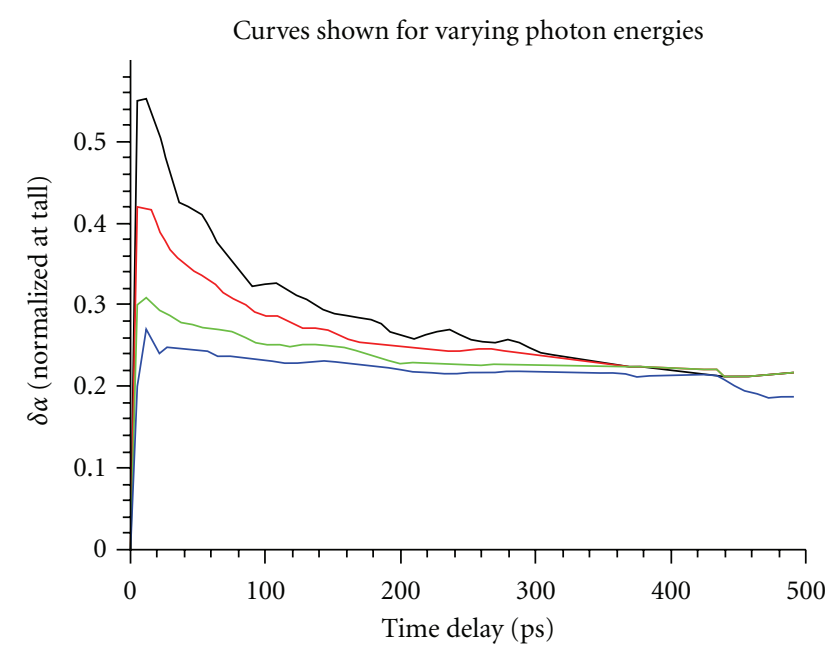

FIGURE 4: Transient spectroscopy signal decay curve [25].

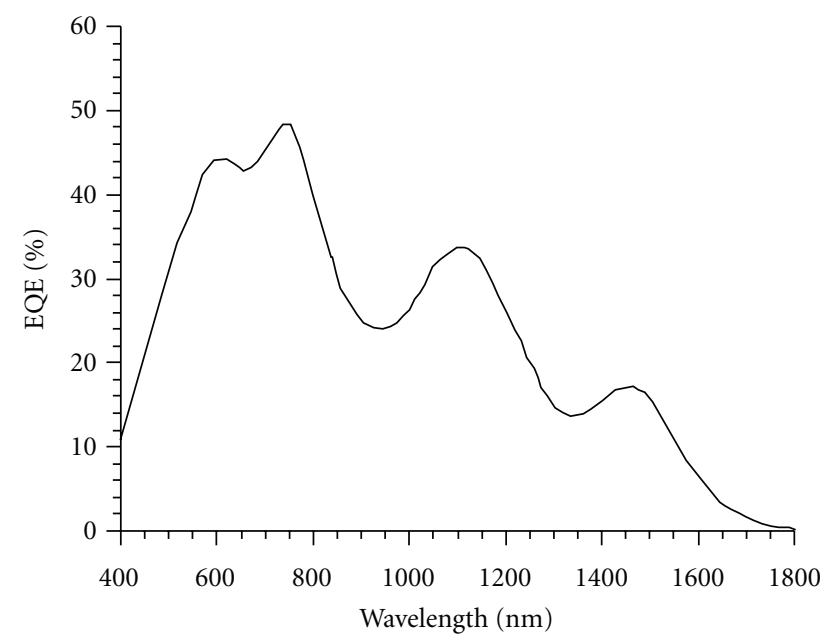

Figure 5: External Quantum Efficiency versus wavelength of quantum dot photo detector [26].

requirement of the processing technology is the variability the process creates in the critical dimension of the nanostructure. Uniformity of the critical dimension inside the device should be maximum for a process to be considered suitable. Figure 6 shows two Gaussian distributions and their full width at half mean (FWHM). Distribution A is the desired case with a small value for FWHM however, because of process variations, the resulting distribution of the manufactured critical dimensions looks like Distribution B. This variation is difficult to optimize, and this arises because of the manufacturing process. From this perspective, the above-mentioned manufacturing techniques are examined in this section.

6.1. Top-Down Approach. Lithography has been the standard technique for transferring patterns during IC manufacturing since its beginning. The minimum feature size, quantified as half pitch, has been steadily decreasing since the 1980s. Invention of better light sources and improved methods of

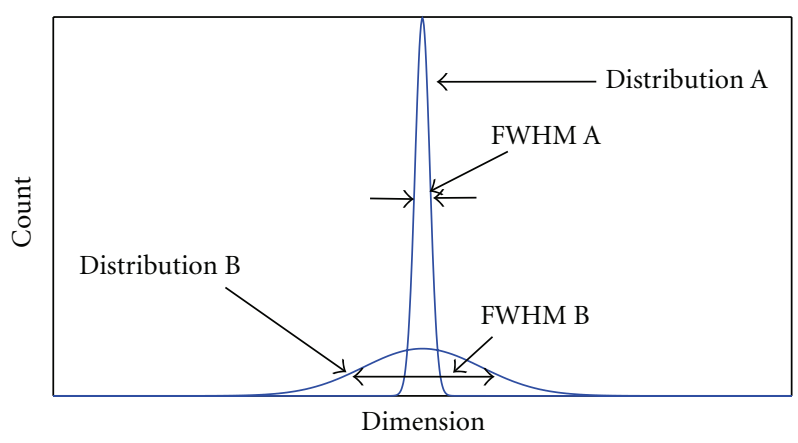

FIgURE 6: Gaussian distributions of the critical dimension.

exposure have driven this change. As of now, the half-pitch distance is $22 \mathrm{~nm}$ and the light source used has a wavelength of $193 \mathrm{~nm}$, which is about nine times the half pitch distance [27]. Further decrease in the light source wavelength to $13.5 \mathrm{~nm}$ using an extreme ultra violet (EUV) source, has the possibility to decrease the half pitch to the range of a few nanometers. Such a trend is shown in Figure 6 [28]. With lithography, patterns of half-pitch distance less than $10 \mathrm{~nm}$ have been created over a decade ago [29]. Two methods are commonly used to do the top-down lithography-one is selective epitaxy, which is growing material on the required areas; the other one is selective etching, which is growing material over a large area and etching out the unnecessary portions to create required structures. Both these methods have been used successfully to create nanostructures.

Apart from surface patterning abilities, manufacturing will require profiling abilities along the vertical axis. The deep reactive ion etching (DRIE) process is currently able to make vertical profiles with aspect ratios greater than 50 . Thus, the traditional, semiconductor industry, based manufacturing technology can make nanodimensional structures for research purposes. However, issues relating to nonhomogeneity and process control will determine if these devices can be successfully manufactured on a large scale without defectrelated problems. Recently, a defect on one of Intel's chip was discovered and analysts predict that this defect is going to cost Intel about \$1 billion [30]. Defects such as these will determine how successful a nanostructure-based product will be. In addition, the high cost of lithography equipment used in IC industry will require major cost reduction, if ever this approach is used by the photovoltaic industry.

6.2. Bottom-Up Approach. The bottom-up approach involves devices using an atom-by-atom approach and is called selfassembly. In our opinion, the meanings of "self-assembly" have been taken wrongly. True self-assembly process involves programmed cell death or apoptosis [31]. The so called "selfassembly" is actually selective chemistry. The atoms or molecules are forced by chemical, mechanical, or electrical means to assemble in a particular fashion. Researchers have often compared this method to the method employed in the development of an animal or a plant. To demonstrate the fundamental problems associated with "self-assembly," we consider the growth of carbon nanotubes (CNTs). Scanning 
tunneling microscope (STM) is used to select multiwall CNT of desired length and diameter [32]. Even with the use of STM, only multiwall CNTs of radius $21 \pm 3 \mathrm{~nm}$ can be obtained [32]. These results are not comparable to the lithography results in terms of variability. In a previous publication [33], we have investigated the basic nature of biodriven systems and shown that due to their fundamental nature of low growth rates as well as their high-defect densities, it is highly unlikely that such systems can be used in semiconductor manufacturing.

In other fields, such as quantum dot lasers, researchers have shown that lithography-based fabrication process gives much better process control and results in structures with better homogeneity than a process using self-assembly [34]. In a controlled experiment involving etching of silicon to form $3 \mathrm{D}$ wire structure to find better photovoltaic properties, researchers found that the maximum efficiency obtained was only $7 \%$ [35]. These are very recent experiments that prove that high efficiency is simply not possible using bottom-up techniques.

\section{Examination of Published Results}

In Table 1, we have included efficiencies that were reported in literature on solar cells that employ the proposed concepts. In some experiments, complicated fabrication methods were used and data from transient spectroscopy or electroluminescence experiments are reported, but neither the $I-V$ data nor the overall efficiency is mentioned. This trend leads the reader away from the reality by talking only about the carrier generation mechanisms. Unless these generated carriers can be separated and collected, there is no solar cell action. As we predicted in 2009 [5], a recently published paper [36] also indicated that a carrier multiplication (CM) process (a process thought to enhance solar cell efficiency) does not take place and the results suggest no improvement in the CM process in nanomaterials in comparison to bulk materials [36].

Thus, as can be observed from Table 1, none of the proposed concepts results in an efficiency increase in the process of converting photo energy to electrical energy when compared to the bulk material solar cell. There is no high efficiency observed in any solar cell as predicted by various theoretical and experimental works.

\section{On the Issue of Process Variations and Defects in Next-Generation Solar Cells}

PV industry has many similarities with the integrated circuit (IC) and light emitting diode (LED) industries. However, there is one important factor that is different. In an IC, one can increase redundancy to account for devices lost because of defects. This is routinely done in the memory industry and results in increased yield and lower fabrication cost. However, this generates an overhead of allocating chip space for memory cells that do not add to the total memory locations and extra logic to detect and replace the faulty devices [37]. In case of solar cells, until now, every cell that is built is
TABLE 1: Summary of experimental results.

\begin{tabular}{lcc}
\hline Cell type & Efficiency & Ref. \\
\hline GaAsNi IBSC & $0.18 \%$ & {$[22]$} \\
GaAs QD IBSC & No data & {$[23]$} \\
Si cell with quantum dots & $5.7-10.6 \%$ & {$[24]$} \\
Excitonic PbSe NC & $3.4 \%$ & {$[38]$} \\
Colloidal PbS QD & $6.0 \%$ & {$[39]$} \\
PbS NC & $4 \%$ & {$[40]$} \\
SL GaAs hot carrier & $10.9 \%-11.2 \%$ & {$[41]$} \\
MQW GaAs hot carrier & $7.1 \%$ & {$[41]$} \\
IBSC QD GaAs & $9.3 \%$ & {$[42]$} \\
Organic solar cell & $8.30 \%$ & {$[7]$} \\
Dye-sensitized solar cell & $10.90 \%$ & {$[7]$} \\
\hline
\end{tabular}

QD: quantum dots; SL: super lattice; NC: nanocrystal.

connected and contributes to the final output power. A defect in any of the cell will result in the deterioration of power output from every cell connected to the cell with defects. The same principle applies to cells that have a lower output voltage or a lower output current due to parametric variations. When connected in series, the cell with the lowest output current will become a bottleneck for the whole module, and, when connected in parallel, the cell with the lowest output voltage will control the module voltage. Thus, defects are very unforgiving in solar cells, and solar cell designs using nanomaterials cannot function unless the defect issue is first solved. A similar approach of including redundant cells in a module can be used; however, the overheads will be somewhat expensive because of the lost real estate.

To illustrate the above mentioned point, a hypothetical case is presented. In Figure 8, individual cells ( $m \times n$ matrix) are connected in series and parallel to fabricate the resultant solar module. $V$ and $J$ are the voltage and current at the maximum power point. Cells in each row are connected in series, and each row is finally connected in parallel with each other. Thus, each row produces a voltage, $V_{1}$, which is the sum of individual voltages, that is, $V_{11}+V_{12}+\cdots+V_{1 n}$, and a current, which is the minimum current in that row, say $J(\mathrm{~min})$. When such rows are connected in parallel, the resulting voltage will be the lowest of the total voltages in each row, $V$ ( $\mathrm{min})$, and the minimum currents of each row will add up to give the final current, $J$ (total). The maximum obtainable current and voltage is thus reduced due to series and parallel connections.

In case of single crystal Si solar cells, open circuit voltage, short circuit current density, and efficiency show Gaussian distribution [43]. Along the line of observed distribution in Si solar cells, very simple calculations were performed. The mean value of the current at the peak power point was assumed to be $25 \mathrm{~mA}$ and that of the voltage was assumed to be $0.5 \mathrm{~V}$. Calculations were done with the 3 sigma variation in voltage and current to be $10 \%, 20 \%, 40 \%$, and $80 \% .100$ such cells were assumed to be connected as 4 blocks in parallel, with each block consisting of 25 cells in series. This arrangement generates about $90 \mathrm{~mA}$ and $12 \mathrm{~V}$, which results 


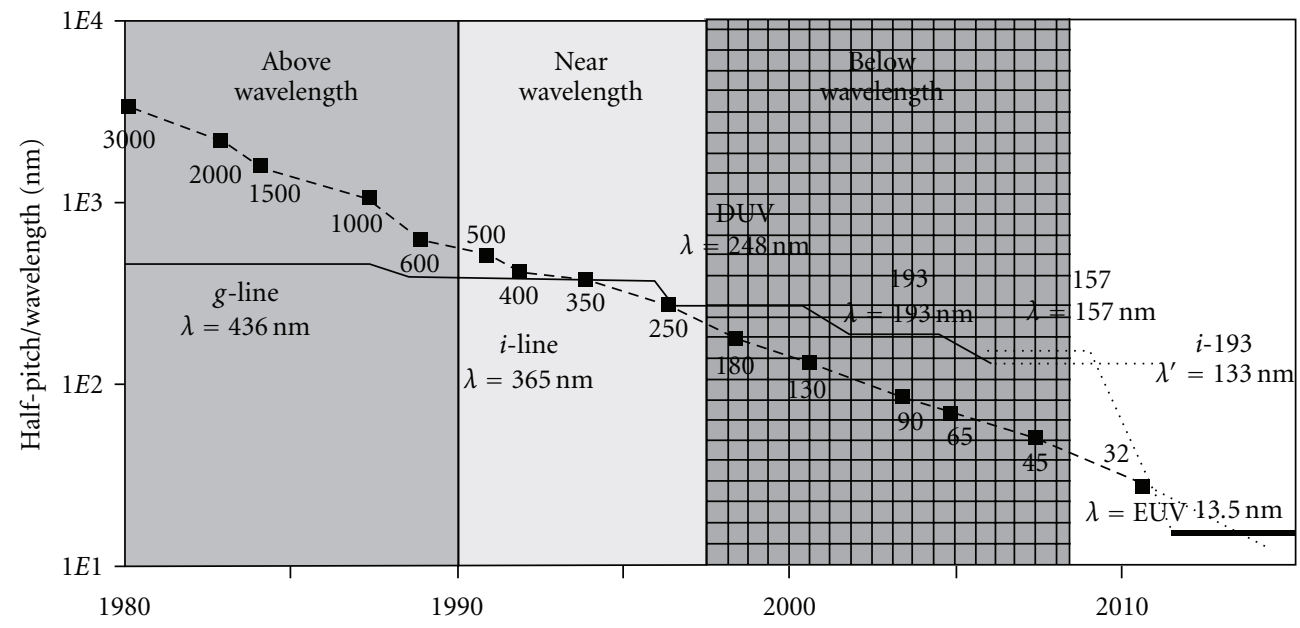

S. Kundu and A. Sreedhar, nanoscale CMOS VLSI circuits: design for manufacturability, McGraw-Hill, 2010 (reproduced with permission).

FIgURE 7: Trend in lithography [28].

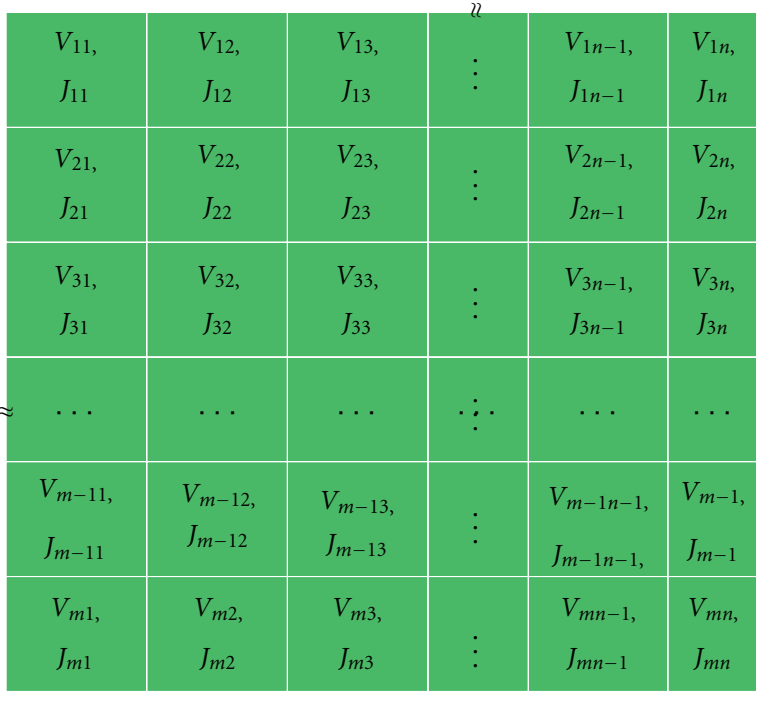

Figure 8: An $m \times n$ array of nanostructured cells connected to form a solar cell.

in a $1.1 \mathrm{~W}$ output power. Random normal data between the 3 sigma points on each side of the mean were generated using the function "normrnd()" on GNU octave for both voltage and current [44]. From the analysis of this data, we come to the conclusion that as the variability in process parameters increases, a significant portion of the power generated is lost in the process of joining cells in series and parallel. On the other hand, individually adding up the power generated in each cell without considering the effect of connections results in only minor loss of power even when variability is high. Practically, to meet certain voltage and current requirements, some sort of connection between cells is required, and this will always result in significant power loss if variability is high. In Figure 7, the results of the above analysis are plotted.

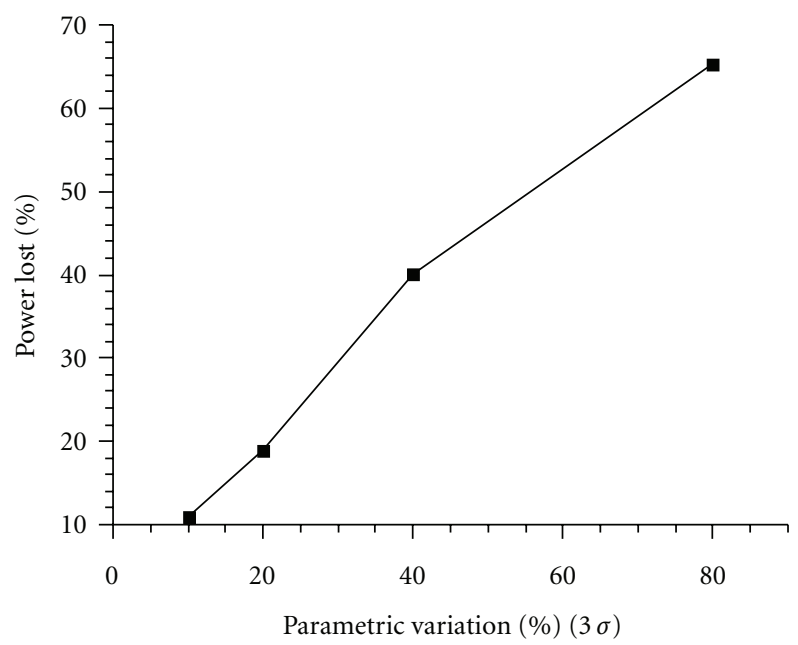

Figure 9: Percentage of power loss as a function of parametric variation.

As can be observed, there is about 55\% decrease in output power as the process variability is increased from $10 \%$ to $80 \%$.

These calculations were performed without considering the effect of reliability and testing issues. When these issues are included, the percentage power lost will be even higher. Other factors such as losses arising due to the interconnects are also ignored to get the final result. In reality, one can expect to see more power losses than shown in Figure 9.

To generalize, also as mentioned in a previous section, the reduction of FWHM is crucial for obtaining the minimum possible variation of critical dimension of the nanostructurebased photovoltaic device. The relationship between FWHM and solar cell efficiency is graphically shown in Figure 10; a very good control on the process will result in devices with 


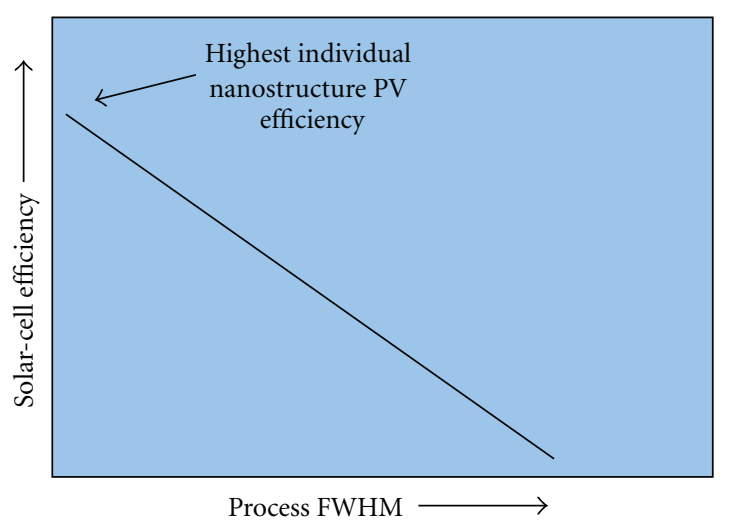

FIGURE 10: Relationship between FWHM and nanostructure-based solar-cell efficiency.

consistent properties and will result in a higher overall cell efficiency.

\section{Discussions}

In the light of the evidences mentioned above, the bottomup approach will not result in useful nanostructures because of lack of control on variability and homogeneity. Quantum physics indeed predicts that the band gap of a material will change as a result of quantization. And, if properly controlled, a structure can be built with materials with varying band gaps. Such a structure can in theory boost the efficiency of a solar cell because the cell will be capable of collecting photons across the complete solar spectrum. This knowledge is not new and has been around for many years. The problem in making such a structure is that a process to create such a structure with the required precision does not exist

If a nanostructured device needs to be manufactured, one is forced to resort to lithography techniques. Extreme ultraviolet- (EUV-) based lithography has the potential of reaching sub-10 $\mathrm{nm}$ resolution. E-beam and ion-beam lithography are proven technologies to create devices in the range of a few nanometers [45]. Tightest process control can be achieved using e-beam lithography, and researchers are making nanostructures with reasonable control regularly. However, this is an expensive and time-consuming process and is not suitable for the production of solar cells on a large scale.

In addition, the simple statistical-process-control- (SPC-) based approach in processing tool should be replaced with the advance-process-control- (APC-) based approach. APC is fundamentally different from SPC, because APC relies on a dynamic-model-based approach to reduce process variability by conducting in situ measurements during the run time. Real-time analysis of such measured data can detect variations in processing conditions that can result in excess variability. This data is used to automatically perform corrective actions to keep the process continuously optimized for the desired results. It should be noted that APC is the current technique used to manufacture many parts of the latest generation of ICs by the semiconductor industry [46]. Unless there is a very tight process control, there is no hope of getting a good efficiency in new generation solar cells. A target for the desired process variability is $10 \%$ at three standard deviations, which is also the standard followed by the IC industry.

The in situ measurements for the APC should be highly precise, because the performance of the manufactured device depends on how accurately the process can be controlled; the input to this control is simply the in situ measurements. New measurement techniques that use quantum effects have been discussed [47]. These measurements schemes are still in their infancy, and further research needs to be conducted in this area to create the extremely precise measurement technique. However, this method of using APC along with ultra lowcost nanodimension lithography is the better option than the bottom-up-based approach. Equipment that will meet the requirements of photovoltaic industry for manufacturing nanostructure-based solar cells does not exist and needs to be invented.

From a market standpoint, one of the barriers for extraordinary growth of the PV industry, similar to that of the mobile phone industry, is that investors are constantly being bombarded with vague claims from researchers that their work will lead to high-efficiency PV cells. This makes the investors cautious in investing in a current manufacturing technology that may be obsolete soon [48]. As an example, the publication of a paper on multiple exciton generation (MEG) in colloidal silicon nanocrystals [49] motivated the author of [50] to claim that silicon nanocrystals-based solar cells can generate two electrons from one photon and that single-junction PV cell efficiency can be as high as $40 \%$. The fact is that the authors of [49] never fabricated a PV device and MEG phenomena reported in nanocrystals or quantum dots have no direct relevance to the operation of the PV cell [5].

\section{Conclusions}

In this paper, we have presented the potential of manufacturing photovoltaic devices beyond current generation of bulk and thin-film semiconductors. Based on the theoretical and experimental results presented, it is obvious that the current processing techniques are unable to capitalize on the advantageous features of nanostructures. New processing methods need to be invented that can provide better dimensional control than existing self-assembly technique. At the same time, the cost of ownership for the new equipment must be lower than that of the tools used presently in the manufacturing of solar cells. Concepts proposed to be used in photovoltaic devices such as multiple exciton generations, carrier multiplication, hot carrier extraction, and intermediate band solar cells have fundamental flaws. Inaccurate assumptions used in the operations of above-mentioned solar cells do not give due importance to transport of photogenerated minority carriers, which is an extremely important process in the operation of a solar cell. In addition, there is no experimental evidence that such "third-generation" devices can perform better than devices based on the current generation of photovoltaic devices. As of now, continuous improvements in bulk and thin-film solar cell manufacturing processes are driving 
costs down and increasing reliability, making solar cells a viable option for power generation.

\section{References}

[1] T. Takamoto, T. Agui, A. Yoshida et al., "World's highest efficiency triple-junction solar cells fabricated by inverted layers transfer process," in Proceedings of the 35th IEEE Photovoltaic Specialists Conference (PVSC '10), pp. 412-417, June 2010.

[2] M. A. Green, "Third generation photovoltaics: ultra-high conversion efficiency at low cost," Progress in Photovoltaics, vol. 9, no. 2, pp. 123-135, 2001.

[3] C. Cui and D. Mattioli, "No silver lining left for users of the metal," The Wall Street Journal, April 2011.

[4] R. Singh and J. D. Leslie, "Economic requirements for new materials for solar photovoltaic cells," Solar Energy, vol. 24, no. 6, pp. 589-592, 1980.

[5] R. Singh, N. Gupta, G. F. Alapatt, R. Podila, and K. F. Poole, "Prospects of nanostructure-based solar cells for manufacturing future generations of photovoltaic modules," International Journal of Photoenergy, vol. 2009, Article ID 154059, 13 pages, 2009.

[6] R. Singh, "Why silicon is and will remain the dominant photovoltaic material," Journal of Nanophotonics, vol. 3, Article ID 032503, 2009.

[7] M. A. Green, K. Emery, Y. Hishikawa, W. Warta, and E. W. Dunlop, "Solar cell efficiency tables (version 38)," Progress in Photovoltaics, vol. 19, no. 1, pp. 565-572, 2011.

[8] M. Jørgensen, K. Norrman, and F. C. Krebs, "Stability/degradation of polymer solar cells," Solar Energy Materials and Solar Cells, vol. 92, no. 7, pp. 686-714, 2008.

[9] K. Kawano, R. Pacios, D. Poplavskyy, J. Nelson, D. D. C. Bradley, and J. R. Durrant, "Degradation of organic solar cells due to air exposure," Solar Energy Materials and Solar Cells, vol. 90, no. 20, pp. 3520-3530, 2006.

[10] P. Buffat and J. P. Borel, "Size effect on the melting temperature of gold particles," Physical Review A, vol. 13, no. 6, pp. 2287 2298, 1976.

[11] R. Singh, P. Chandran, M. Grujicic et al., "Dominance of silicon CMOS based semiconductor manufacturing beyond international technology roadmap and many more decades to come," in Semiconductor Fabtech, pp. 104-113, 30th edition, 2006.

[12] V. V. Pokropivny and V. V. Skorokhod, "Classification of nanostructures by dimensionality and concept of surface forms engineering in nanomaterial science," Materials Science and Engineering C, vol. 27, no. 5-8, pp. 990-993, 2007.

[13] J. Henk, Introduction to the Theory of Nanostructures, International Max Planck Research School on Science and Technology of Nanostructures, 2006.

[14] W. Shockley and H. J. Queisser, "Detailed balance limit of efficiency of p-n junction solar cells," Journal of Applied Physics, vol. 32, no. 3, pp. 510-519, 1961.

[15] R. T. Ross and A. J. Nozik, "Efficiency of hot-carrier solar energy converters," Journal of Applied Physics, vol. 53, no. 5, pp. 3813-3818, 1982.

[16] A. Luque and A. Martí, "Increasing the efficiency of ideal solar cells by photon induced transitions at intermediate levels," Physical Review Letters, vol. 78, no. 26, pp. 5014-5017, 1997.

[17] A. J. Nozik, "Quantum dot solar cells," Physica E, vol. 14, no. 1-2, pp. 115-120, 2002.
[18] S. Kolodinski, J. H. Werner, T. Wittchen, and H. J. Queisser, "Quantum efficiencies exceeding unity due to impact ionization in silicon solar cells," Applied Physics Letters, vol. 63, no. 17, pp. 2405-2407, 1993.

[19] A. Shabaev, A. L. Efros, and A. J. Nozik, "Multiexciton generation by a single photon in nanocrystals," Nano Letters, vol. 6, no. 12, pp. 2856-2863, 2006.

[20] M. Wolf, "Limitations and possibilities for improvement of photovoltaic solar energy converters: part I: considerations for earth's surface operation," Proceedings of the IRE, vol. 48, pp. 1246-1263, 1960.

[21] A. J. Nozik, "Exciton multiplication and relaxation dynamics in quantum dots: applications to ultra-high efficiency solar photon conversion," in Proceedings of the 4th IEEE World Conference on Photovoltaic Energy Conversion (WCPEC '06), pp. 40-44, May 2006.

[22] V. I. Klimov, "Mechanisms for photogeneration and recombination of multiexcitons in semiconductor nanocrystals: implications for lasing and solar energy conversion," Journal of Physical Chemistry B, vol. 110, no. 34, pp. 16827-16845, 2006.

[23] C. M. Isborn, S. V. Kilina, X. Li, and O. V. Prezhdo, "Generation of multiple excitons in PbSe and CdSe quantum dots by direct photoexcitation: first-principles calculations on small PbSe and CdSe clusters," Journal of Physical Chemistry C, vol. 112, no. 47, pp. 18291-18294, 2008.

[24] P. V. Kamat, "Quantum dot solar cells. Semiconductor nanocrystals as light harvesters," Journal of Physical Chemistry C, vol. 112, no. 48, pp. 18737-18753, 2008.

[25] R. J. Ellingson, M. C. Beard, J. C. Johnson et al., "Highly efficient multiple exciton generation in colloidal $\mathrm{PbSe}$ and $\mathrm{PbS}$ quantum dots," Nano Letters, vol. 5, no. 5, pp. 865-871, 2005.

[26] G. Konstantatos and E. H. Sargent, "Solution-processed quantum dot photodetectors," Proceedings of the IEEE, vol. 97, no. 10, Article ID 5247124, pp. 1666-1683, 2009.

[27] R. Merritt, "Intel details its first $22 \mathrm{~nm}$ processer," http://www .eetasia.com/ART_8800651608_499489_NT_066e1853.HTM.

[28] S. Kundu and A. Sreedhar, Nanoscale CMOS VLSI Circuits: Design for Manufacturability, McGraw-Hill, New York, NY, USA, 2010.

[29] S. Y. Chou, P. R. Krauss, W. Zhang, L. Guo, and L. Zhuang, "Sub-10 nm imprint lithography and applications," Journal of Vacuum Science and Technology B, vol. 15, no. 6, pp. 28972904, 1997.

[30] D. Clark and S. Tibken, "Intel finds chip-design flaw," The Wall Street Journal, February 2011.

[31] M. T. Heemels, R. Dhand, and L. Allen, "Foreword: apoptosis," Nature, vol. 407, pp. 767-770, 2000.

[32] O. Loh, X. Wei, C. Ke, J. Sullivan, and H. D. Espinosa, "Robust carbon-nanotube-based nano-electromechanical devices: understanding and eliminating prevalent failure modes using alternative electrode materials," Small, vol. 7, no. 1, pp. 79-86, 2011.

[33] R. Singh, T. Boland, and R. Mulye, "Prospects of incorporating directed self assembly into semiconductor manufacturing," in Semiconductor Fabtech, vol. 36, pp. 67-72, 36th edition, 2007.

[34] J. J. Coleman, J. D. Young, and A. Garg, "Semiconductor quantum dot lasers: a tutorial," Journal of Lightwave Technology, vol. 29, no. 4, pp. 499-510, 2011.

[35] S. H. Baek, H. S. Jang, and J. H. Kim, "Characterization of optical absorption and photovoltaic properties of silicon wire solar cells with different aspect ratio," Current Applied Physics, vol. 11, pp. S30-S33, 2010.

[36] G. Nair, L.-Y. Chang, S. M. Geyer, and M. G. Bawendi, "Perspective on the prospects of a carrier multiplication nanocrystal solar cell," Nano Letters, vol. 11, no. 5, pp. 2145-2151, 2011. 
[37] M. Horiguchi and K. Itoh, "Redundancy," in Nanoscale Memory Repair, pp. 19-67, Springer, New York, NY, USA, 2011.

[38] N. López, L. A. Reichertz, K. M. Yu, K. Campman, and W. Walukiewicz, "Engineering the electronic band structure for multiband solar cells," Physical Review Letters, vol. 106, no. 2, Article ID 028701, 4 pages, 2011.

[39] J. Tang, K. W. Kemp, S. Hoogland et al., "Colloidal-quantumdot photovoltaics using atomic-ligand passivation," Nature Materials, vol. 10, no. 10, pp. 765-771, 2011.

[40] E. C. Cho, S. Park, X. Hao et al., "Silicon quantum dot/ crystalline silicon solar cells," Nanotechnology, vol. 19, no. 24, Article ID 245201, 2008.

[41] J. J. Choi, Y. F. Lim, M. B. Santiago-Berrios et al., "PbSe nanocrystal excitonic solar cells," Nano Letters, vol. 9, no. 11, pp. 3749-3755, 2009.

[42] N. Zhao, T. P. Osedach, L. Y. Chang et al., "Colloidal PbS quantum dot solar cells with high fill factor," ACS Nano, vol. 4, no. 7, pp. 3743-3752, 2010.

[43] A. Ebong, I. B. Cooper, B. Rounsaville et al., "High efficiency inline diffused emitter (ILDE) solar cells on mono-crystalline CZ silicon," Progress in Photovoltaics, vol. 18, no. 8, pp. 590$595,2010$.

[44] J. W. Eaton, GNU Octave Manual, Network Theory Limited, 2002.

[45] W. F. van Dorp, I. Lazić, A. Beyer et al., "Ultrahigh resolution focused electron beam induced processing: the effect of substrate thickness," Nanotechnology, vol. 22, no. 11, Article ID 115303, 2011.

[46] P. McGuire and A. Mullins, "Key aspects of advanced process control," Future Fab International, vol. 4, no. 1, 1998.

[47] V. Giovannetti, S. Lloyd, and L. MacCone, "Advances in quantum metrology," Nature Photonics, vol. 5, no. 4, pp. 222229, 2011.

[48] R. Singh, Sustainable Energy for Sustainable Economic Growth, Forthcoming Book, 2012.

[49] M. C. Beard, K. P. Knutsen, P. Yu et al., "Multiple exciton generation in colloidal silicon nanocrystals," Nano Letters, vol. 7, no. 8, pp. 2506-2512, 2007.

[50] K. Bullis, "Nanocrystals for Superefficient Solar Cells," Technology Review, March 2007, http://www.technologyreview .com/energy/19256. 

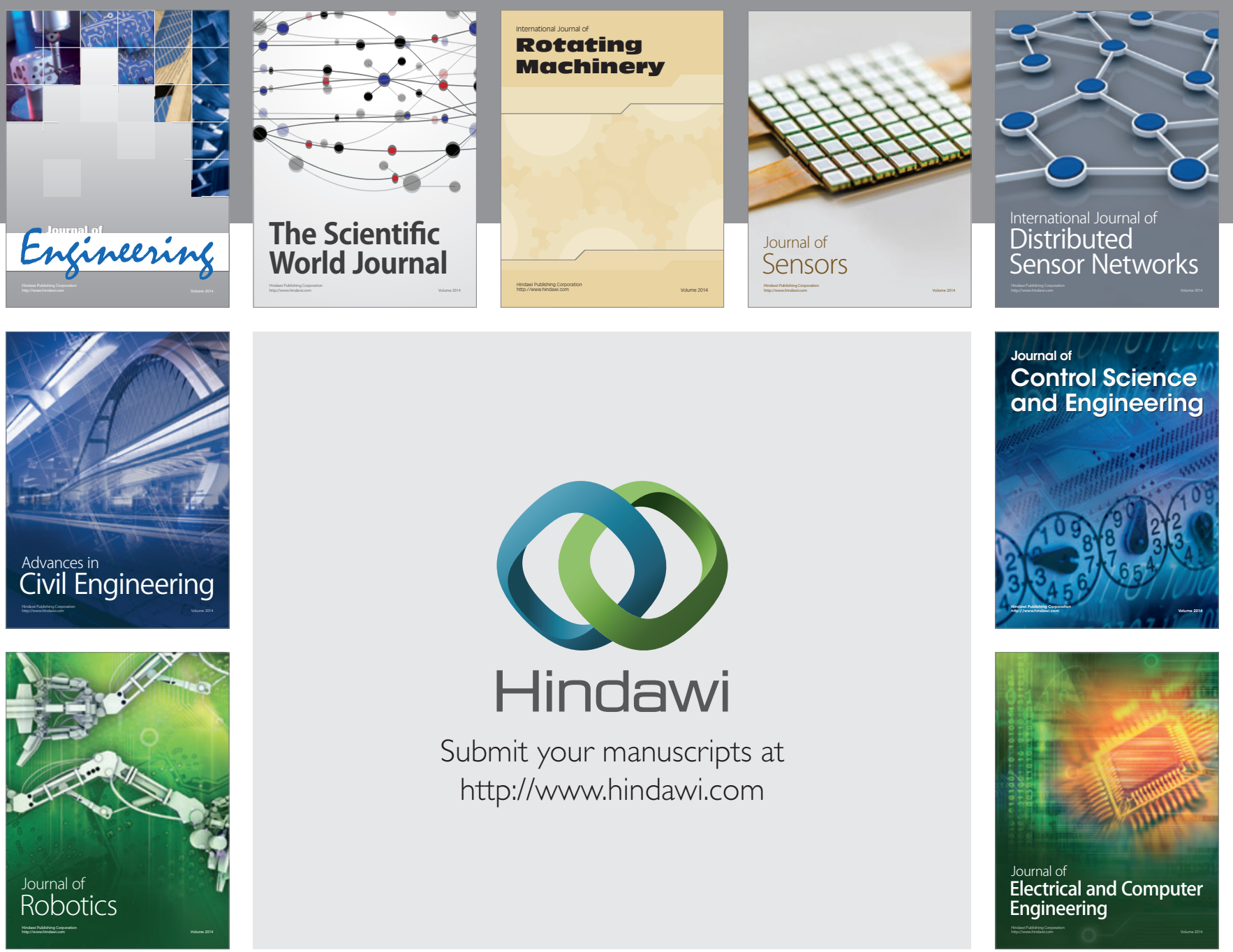

Submit your manuscripts at

http://www.hindawi.com
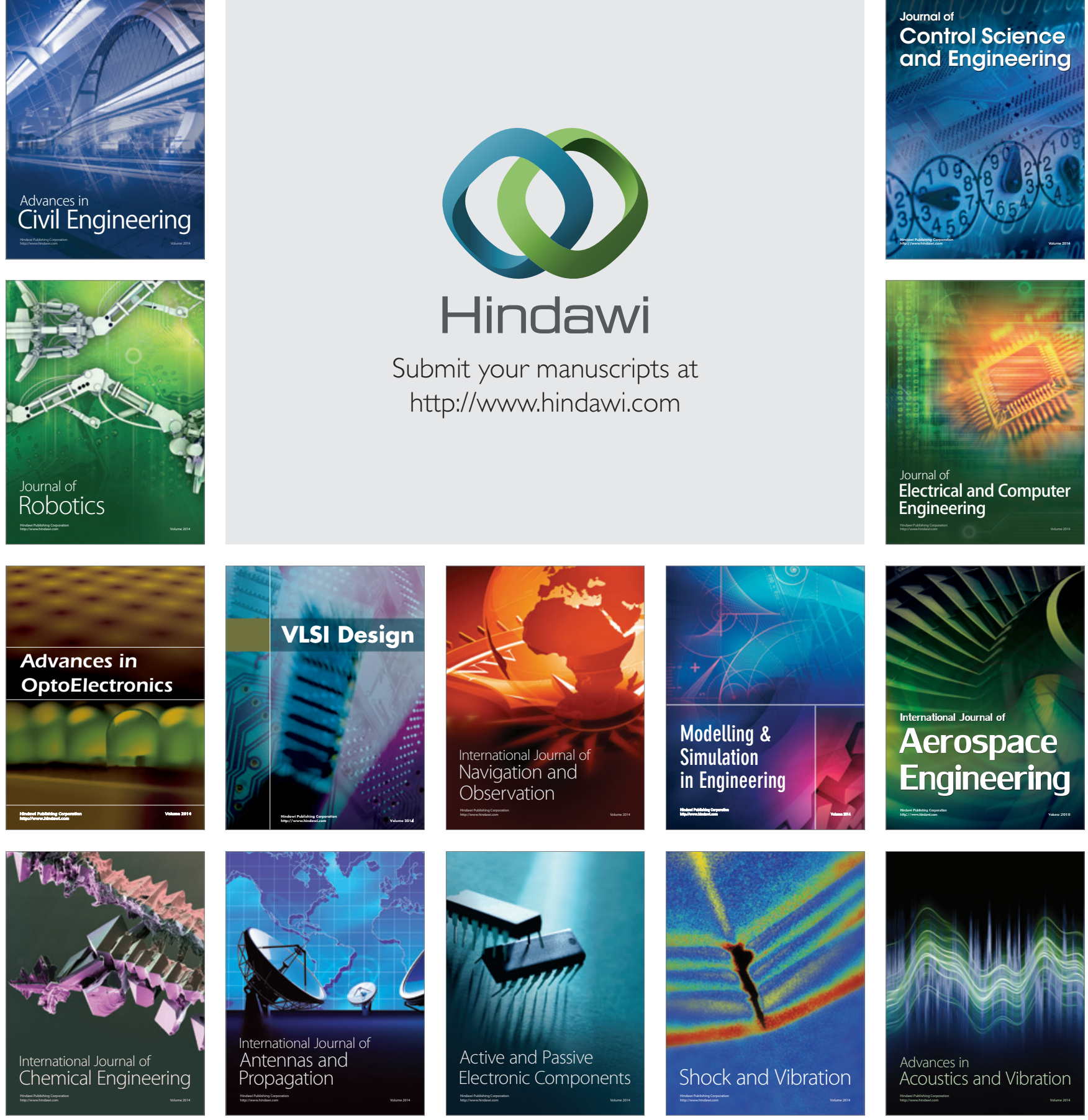